\title{
Statistical Template Matching under Geometric Transformations
}

\author{
Alexander Sibiryakov \\ Mitsubishi Electric ITE-BV, Guildford, United Kingdom \\ Alexander.Sibiryakov@vil.ite.mee.com
}

\begin{abstract}
We present a novel template matching framework for detecting geometrically transformed objects. A template is a simplified representation of the object of interest by a set of pixel groups of any shape, and the similarity between the template and an image region is derived from the F-test statistic. The method selects a geometric transformation from a discrete set of transformations, giving the best statistical independence of such groups Efficient matching is achieved using 1D analogue of integral images - integral lines, and the number of operations required to compute the matching score is linear with template size, comparing to quadratic dependency in conventional template matching. Although the assumption that the geometric deformation can be approximated from discrete set of transforms is restrictive, we introduce an adaptive subpixel refinement stage for accurate matching of object under arbitrary parametric 2D-transformation. The parameters maximizing the matching score are found by solving an equivalent eigenvalue problem. The methods are demonstrated on synthetic and real-world examples and compared to standard template matching methods.
\end{abstract}

\section{Introduction}

Template matching (TM) is a standard computer vision tool for finding objects or object parts in images. It is used in many applications including remote sensing, medical imaging, and automatic inspection in industry. The detection of real-word objects is a challenging problem due to the presence of illumination and color changes, partial occlusions, noise and clutter in the background, and dynamic changes in the object itself.

A variety of template matching algorithms have been proposed, ranging from extremely fast computing simple rectangular features $[1,2]$ to fitting rigidly or non-rigidly deformed templates to image data $[3,4,13]$.

The exhaustive search strategy of template matching is the following: for every possible location, rotation, scale, or other geometric transformation, compare each image region to a template and select the best matching scores. This computationally expensive approach requires $\mathrm{O}\left(N_{l} N_{g} N_{t}\right)$ operations, where $N_{l}$ is the number of locations in the image, $N_{p}$ is the number of transformation samples, and $N_{t}$ is the number of pixels used in matching score computation. Many methods try to reduce the computational complexity. $N_{l}$ and $N_{g}$ are usually reduced by the multiresolution approach (e.g.,[4]) or by projecting the template and image patches onto a rotation-invariant basis [12], but while excluding rotation, these projection-based methods still need a strategy of scale selection. Often the geometric transformations are not included in the matching strategy at all, assuming that the template and the image patch differ by translation only [11]. 
Another way to perform TM is direct fitting of the template using gradient descent or ascent optimization methods to iteratively adjust the geometric transformation until the best match is found [10]. These techniques need initial approximations that are close to the right solution.

In rapid TM methods $[1,2,5,6,7]$, the term $N_{t}$ in the computational complexity defined above is reduced by template simplification, e.g., by representing the template as a combination of rectangles. Using special image preprocessing techniques (so-called integral images) and computing a simplified similarity score (the normalized contrast between "positive" and "negative" image regions defined by the template), the computat- ional speed of rapid TM is independent of the template size and depends only on the template complexity (the number of rectangles comprising the template). However, such Haar-like features are not rotation-invariant, and a few extensions [5-7] of this framework have been proposed to handle the image rotation. [5] proposed additional set diagonal rectangular templates. [6] proposed $45^{\circ}$ twisted Haar-like features computed via $45^{\circ}$ rotated integral images. [7] further extended this idea and used multiple sets of Haar-like features and integral images rotated by whole integer-pixel based rotations.

Rapid TM framework has a few implicit drawbacks, which are not presented in computationally expensive correlation-based TM methods:

- It is not easy to generalize two-region Haar-like features to the case of three or more pixel groups.

- Rectangle-based representation is redundant for curvilinear object shapes, e.g. circles. Usage of the curved templates instead of the rectangular ones should result in such cases in higher matching scores and, therefore, in better detector performance.

- Impressive results with Haar-like features were achieved by using powerful classifiers based on boosting [1]. They require training on large databases and, therefore, matching using single object template (achievable at no additional cost in correlation-based TM using grayscale template) cannot be easily performed in this framework, or it can be performed only for objects having simple shape and bimodal intensity distribution.

This paper proposes a new approach that can be placed in between rapid TM methods and standard correlation-based TM methods. The proposed approach solves all limitations listed above and can also be extended to an iterative refinement framework for precise estimation of object location and transformation. The method is based on Statistical Template Matching (STM), first introduced in [2]. STM framework is very similar to rapid TM framework discussed above; the main difference is that STM uses a different matching score derived from the F-test statistic, supporting multiple pixel groups. The STM method is overviewed in Section 2. Section 3 presents a new extension of STM for rotated and scaled objects based on integral lines. Although 1D integral lines technique may seem an obvious and particular case of 2D integral images, the area of applicability of the proposed template matching is much wider when using integral lines, than when using integral images. The integral images technique requires object shape combined of rectan- gles, whereas integral lines method requires just a combination of line segments, which is obviously more general case, because any rasterized $2 \mathrm{D}$ shape can be represented as a combination of segments. Section 4 presents another new extension, Adaptive Subpixel (AS) STM, suitable for accurate estimation of parametric 2D-transformation of the object. An efficient solution for a particular case of Haar-like templates is given. Section 5 demonstrates the methods in a few computer vision tasks. Section 6 concludes the paper. 


\section{Statistical Template Matching}

The name Statistical Template Matching originates from the fact that only statistical characteristics of pixel groups, such as mean and variance, are used in the analysis. These pixel groups are determined by a topological template, which is the analogue of the Haarlike feature in a two-group case. The topological template is a set of $N$ regions $T_{0}=T_{1} \mathrm{U} \ldots \mathrm{U} T_{N}$, representing spatial relation of object parts. Each region $T_{i}$ may consist of disconnected sub-regions of arbitrary shape. If image pixel groups, defined by template regions, statistically differ from each other, it is likely that these pixel groups belong to the object of interest. This principle can be demonstrated by a simplified example shown in Fig.1, where the template $T_{0}=T_{1} \cup T_{2} \mathrm{U} T_{3}$ is matched to image regions $\mathrm{R}_{1}$ and $\mathrm{R}_{2}$. In the first case, three pixel groups are similar, as they have roughly the same mean value. In the second case, the pixel groups are different (black, dark-gray and light-gray mean colours), from which we conclude that $\mathrm{R}_{2}$ is similar to the template.
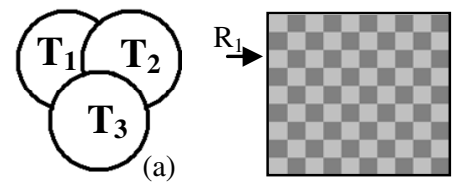

(b)
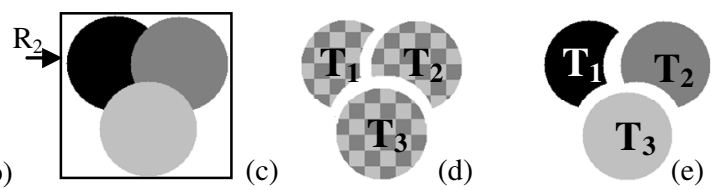

Fig. 1. Simplified example of STM: (a) A template consisting of three regions of circular shape; (b) $1^{\text {st }}$ region of interest $\left(R_{1}\right)$ in an image; (c) $2^{\text {nd }}$ region in the image $\left(R_{2}\right)$; $(d)$ Decomposition of $R_{1}$ into three regions by the template: pixel groups are similar; (e) Decomposition of the $\mathrm{R}_{2}$ : pixel groups are different.

Formally, such a similarity (the matching score) between the template $T_{0}$ and an image region $R(\mathbf{x})$, centered at some pixel $\mathbf{x}=(x, y)$, is derived from the F-test statistic. Denote the number of pixels, mean and variance in the region $T_{i},(i=0, \ldots, N)$ as $n_{i}, m_{i}$ and $\sigma_{i}^{2}$ respectively. Assuming normal distribution of pixel values and equal variances, and using the standard Analysis Of Variances (ANOVA) technique, we define the Between-group variation $V_{B G}$ and Within-group variation $V_{W G}$ :

$$
V_{B G}\left(T_{1}, \ldots, T_{N}\right)=-n_{0} m_{0}^{2}+\sum_{i=1}^{N} n_{i} m_{i}^{2}, \quad V_{W G}\left(T_{1}, \ldots, T_{N}\right)=\sum_{i=1}^{N} n_{i} \sigma_{i}^{2},
$$

Taking into account degrees of freedom of $V_{B G}$ and $V_{W G}$, they relationship $V_{B G}+$ $V_{W G}=n_{0} \sigma_{0}^{2}$ and applying equivalent transformations, the F-variable becomes

$$
F=\frac{V_{B G}}{V_{W G}} \frac{n_{0}-N}{N-1}=\left(\frac{n_{0} \sigma_{0}^{2}}{n_{1} \sigma_{1}^{2}+\ldots+n_{N} \sigma_{N}^{2}}-1\right) \frac{n_{0}-N}{N-1} .
$$

Removing constant terms in (2), we obtain the expression for matching score [2]:

$$
S(\mathbf{x})=\frac{n_{0} \sigma_{0}^{2}}{n_{1} \sigma_{1}^{2}+\ldots+n_{N} \sigma_{N}^{2}} .
$$

Computed for all pixels $\mathbf{x}$, the matching scores (3) form a confidence map, in which the local maxima correspond to likely object locations. Application-dependent analysis of statistics $m_{i}, \sigma_{i}$ helps to reduce the number of false alarms. When photometric properties 
of the object parts are given in advance, e.g., some of the regions are darker or less textured than the others, additional constraints, such as (4), reject false local maxima.

$$
m_{i}<m_{j}, \quad \sigma_{i}<\sigma_{j}
$$

For Haar-like features $(N=2)$, the matching score (3) can also be derived from the squared t-test statistic, which is the squared signal-to-noise ratio (SNR), ranging from 1 (noise), corresponding to the case when all groups are similar, to infinity (pure signal), corresponding to the case when the template strictly determines the layout of pixel groups and all pixels in a group are equal. The distribution of pixel values in image patches can be arbitrary and usually does not satisfy the above assumptions (normal distribution, equal variances); therefore, in practice, it is convenient to interpret (3) as SNR. Instead of using statistical tables for the F-variable, a reasonable SNR threshold above 1 can determine if the similarity (3) between the template and the image region is large enough.

The real-time implementation of STM [2] uses templates with regions $T_{i}$ consisting of the union of rectangles. Using the integral images, the pixel variances from (3) are computed using only $8 k_{i}$ memory references, where $k_{i}$ is a number of rectangles in $T_{i}$.

\section{STM under Geometric Transformations}

In the generalized STM we consider an object of interest transformed by a transformation $\mathbf{P}$ with unknown parameters $\mathbf{p}=\left(p_{1}, \ldots, p_{k}\right)^{\mathrm{T}}$. This is schematically shown in Fig.2. In order to match the object accurately, the template should be transformed using the same model P. As the parameters are unknown, all combinations $\left(p_{1}^{\left(j_{1}\right)}, \ldots, p_{k}^{\left(j_{k}\right)}\right)$ of their discrete values $p_{i}^{(j)}=p_{i \min }+j \Delta p_{i}$ are used to transform the template and compute the best matching score:

$$
S(\mathbf{x})=\max _{p_{1}, \ldots, p_{k}} S\left(\mathbf{x} ; p_{1}, \ldots, p_{k}\right)
$$

By storing the indexes of the best parameter combination

$$
\left(j_{1}, \ldots, j_{k}\right)^{*}=\underset{j_{1}, \ldots, j_{k}}{\arg \max } S\left(\mathbf{x} ; p_{1}^{\left(j_{1}\right)}, \ldots, p_{k}^{\left(j_{k}\right)}\right),
$$

it is possible to recover an approximated object pose. The number of parameter com binations and computational time grow exponentially with the number of parameters; therefore, it is essential to use a minimal number of parameters. Many approaches [4$7,12,13]$ use the fact that moderate affine and perspective distortions are approximated well by the similarity transform requiring only two additional parameters for rotation and scale. In our method we also apply a set of similarity transforms to the template and select for each location those rotation and scale parameters giving the best matching score (5)-(6). Although the assumption that the geometric deformations are small enough to be approximated by similarity transform is restrictive, in the next section we describe an iterative technique of recovering full parametric 2D-transformation using similarity transform as initial approximation.

The transformed template is rasterized, and each region is represented by a set of line segments (Fig.3): $T_{i}=\left\{s_{i, j} \mid s_{i, j}=\left(x_{1}, x_{2} y\right)_{i, j}\right\}$. Each segment is a rectangle of one-pixel height, and integral images technique can still be used to compute the variances in (3). This is 
not optimal way of computation, and to handle segments efficiently, we propose a onedimensional analogue of integral images, integral lines, defined as follows:

$$
I_{1}(x, y)=\sum_{a \leq x} f(a, y) ; \quad I_{2}(x, y)=\sum_{a \leq x} f^{2}(a, y)
$$

A similar definition can be given for integral vertical lines, where integration is performed along the $y$ axis. The sums required for computation of the variances in (3), can now be computed via integral lines as follows:

$$
u_{i} \equiv \sum_{(x, y) \in T_{i}} f(x, y)=\sum_{\left(x_{1}, x_{2}, y\right) \in T}\left(I_{1}\left(x_{2}, y\right)-I_{1}\left(x_{1}-1, y\right)\right), \quad v_{i} \equiv \sum_{(x, y) \in T_{i}} f^{2}(x, y)=\sum_{\left(x_{1}, x_{2}, y\right) \in T}\left(I_{2}\left(x_{2}, y\right)-I_{2}\left(x_{1}-1, y\right)\right)
$$

where $I_{1}(-1, y)=I_{2}(-1, y)=0$. Thus, the number of memory references is reduced from the number of pixels to the number of lines in the rasterized template.

For efficient implementation, we rewrite (3) in a more convenient form (9) using definitions (8):

$$
S=\frac{v_{0}-u_{0}^{2} / n_{0}}{v_{0}-\left(\sum_{i=1}^{N-1} \frac{u_{i}^{2}}{n_{i}}\right)-\frac{1}{n_{N}}\left(u_{0}-\sum_{i=1}^{N-1} u_{i}\right)^{2}} .
$$

Thus, the algorithm does not requite multiple sums of squared pixels $v_{i}$ to compute the matching score. It is sufficient to compute only the sum of squared pixels in the entire template $T_{0}$ and $N$ sums of pixels in $T_{0}, T_{1, \ldots,} T_{N-1}$. Moreover, for a rotationally-symmetrical template, $v_{0}$ and $u_{0}$ remain constant for each rotation angle, and only $u_{1}, . ., u_{M-1}$ need recomputing. Excluding one region $T_{N}$ from computations gives additional advantage in computation speed, as we can denote as $T_{N}$ the most complex region, consisting of the largest number of lines. Line configurations change during template rotation, thus alternating the most complex region at each rotation angle.

Rapid STM [2] requires $\Sigma 8 k_{i}$ memory references independently on template size, where $k_{i}$ is a number of rectangles in the region $T_{i}$. Correlation-based TM requires $N_{t}$ (the number of pixels) memory references, quadratically dependent on the template size. In the generalized STM, the number of memory references is $4 k_{0}+2 k_{l}+\ldots+2 k_{N-1}$, where $k_{i}$ is the number of lines in the template region $T_{i}$. The total number of lines is roughly proportional to the template height multiplied by the number of regions $N$; therefore, it depends linearly on template size. Thus, the computational efficiency of the proposed method lies between that of the rapid TM and correlation-based TM methods.

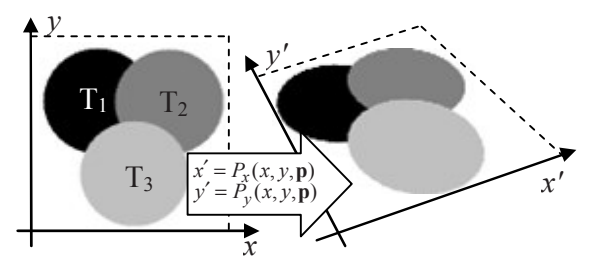

Fig. 2. Example of object transformation (perspective model

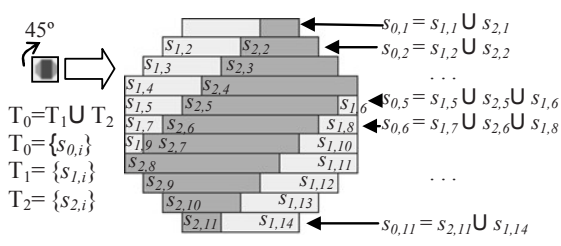

Fig. 3. Rotation of a two-region template by $45^{\circ}$ and its representation by a set of lines 


\section{Adaptive Subpixel STM}

The method proposed in this section is not restricted by rotation and scale only, and uses full transformation $\mathbf{P}$ (Fig.2) to iteratively estimate object location and transformation with high accuracy. In this paper, we use the perspective model for all simulations, but any other parametric transformation is also applicable. The goal of the iterative STM method is to compute transformation parameters $\mathbf{p}$ adaptively from image data, maximizing the matching score $S(\mathbf{x}, \mathbf{p})$ at a particular object location $\mathbf{x}$. The discrete method from Section 3 can be used to find an initial approximation of the object location $\mathbf{x}_{\mathbf{0}}=\left(x_{0}, y_{0}\right)$ and initial transformation parameters $\mathbf{p}_{\mathbf{0}}$. Following the standard technique of iterative image registration [10], we obtain a linear approximation of the transformed pixels $\left(x_{0}^{\prime}, y_{0}^{\prime}\right)$ near their initial location $\left(x_{0}, y_{0}\right)$. Such an approximation is given by

$$
\begin{aligned}
& f^{\prime}\left(x_{0}^{\prime}, y_{0}^{\prime}\right) \approx f\left(x_{0}, y_{0}\right)+\frac{\partial f(x, y)}{\partial x} \Delta x^{\prime}+\frac{\partial f(x, y)}{\partial y} \Delta y^{\prime} \\
& =f\left(x_{0}, y_{0}\right)+\frac{\partial f(x, y)}{\partial x} \sum \frac{\partial x^{\prime}}{\partial p_{i}} \Delta p_{i}+\frac{\partial f(x, y)}{\partial y} \sum \frac{\partial y^{\prime}}{\partial p_{i}} \Delta p_{i} \equiv \mathbf{f}^{T}\left(x_{0}, y_{0}\right) \Delta \mathbf{p},
\end{aligned}
$$

where $\Delta \mathbf{p}=\left(1, \Delta p_{1}, \ldots, \Delta p_{k}\right)^{T}$ is a vector of parameter amendments and

$$
\begin{gathered}
\mathbf{f}^{T}\left(x_{0}, y_{0}\right)=\left(f\left(x_{0}, y_{0}\right), f_{p_{1}}\left(x_{0}, y_{0}\right), \ldots, f_{p_{k}}\left(x_{0}, y_{0}\right)\right) \\
f_{p_{j}}=\frac{\partial f(x, y)}{\partial x} \frac{\partial x^{\prime}}{\partial p_{j}}+\frac{\partial f(x, y)}{\partial y} \frac{\partial y^{\prime}}{\partial p_{j}}
\end{gathered}
$$

From (8), the linearized expressions for $u_{i}^{2}$ and $v_{i}$ have the following matrix form:

$$
\begin{gathered}
v_{i} \approx \Delta \mathbf{p}^{T}\left(\sum_{(x, y) \in T_{i}} \mathbf{f}(x, y) \mathbf{f}^{T}(x, y)\right) \Delta \mathbf{p} \equiv \Delta \mathbf{p}^{T} \mathbf{V}_{\mathbf{i}} \Delta \mathbf{p} \\
\frac{u_{i}^{2}}{n_{i}} \approx \Delta \mathbf{p}^{T} \frac{1}{n_{i}}\left(\sum_{(x, y) \in T_{i}} \mathbf{f}(x, y)\right)\left(\sum_{(x, y) \in T_{i}} \mathbf{f}(x, y)\right)^{T} \Delta \mathbf{p} \equiv \Delta \mathbf{p}^{T} \mathbf{U}_{\mathbf{i}} \Delta \mathbf{p}
\end{gathered}
$$

Substituting (13) and (14) to (9), we obtain the linearized matching score in the form of the Rayleigh quotient:

$$
S=\frac{\Delta \mathbf{p}^{T} \mathbf{A} \Delta \mathbf{p}}{\Delta \mathbf{p}^{T} \mathbf{B} \Delta \mathbf{p}},
$$

where $\mathbf{A}=\mathbf{V}_{\mathbf{0}}-\mathbf{U}_{\mathbf{0}}, \mathbf{B}=\mathbf{V}_{\mathbf{0}}-\mathbf{U}_{\mathbf{1}}-\ldots-\mathbf{U}_{\mathbf{k}}$. The matrices $\mathbf{A}$ and $\mathbf{B}$ are one-rank modifications of the same covariance matrix $\mathbf{V}_{\mathbf{0}}$. They are symmetric by definition and positivedefinite, which follows from the fact that both numerator and denominator in (15) are image variances.

Maximization of the Rayleigh quotient (15) is equivalent to solving a generalized eigenvalue problem

$$
\mathbf{A} \Delta \mathbf{p}=S \mathbf{B} \Delta \mathbf{p},
$$

Any state-of-the-art method from linear algebra can be used to find the largest eigenvalue $S$ (which is also the maximized matching score) and corresponding eigenvector $\Delta \mathbf{p}$ (the amendments to the image transformation parameters). Examples of such methods are power iterations and inverse iterations (see [8] for a detailed review). 
When the eigenvector $\Delta \mathbf{p}$ is found, any vector of the form $\alpha \Delta \mathbf{p}$ is also a solution of (16). Selecting an optimal $\alpha$ that improves the convergence and prevents the solution from oscillations around the maximum is an important part of the algori- thm, and we found that Linesearch strategy provides a robust solution. A detailed review of this and other strategies can be found in [9].

The original non-linear problem can be solved by iteratively applying the linearized solution. The iterations stop when the matching score, the center of the image patch and/or parameter amendments do not change significantly. Below is the outline of the AS STM algorithm that starts at iteration $n=0$ from initial values $S_{0}, \mathbf{x}_{\mathbf{0}}, \mathbf{p}_{\mathbf{0}}$ :

1. Resample image patch centered at $\mathbf{x}_{\mathbf{n}}$ using current $\mathbf{p}_{\mathbf{n}}$

2. Compute image derivatives from resampled image patch $f(x, y)$; compute partial derivatives of the transformation model $\mathbf{P}$ in (12) using current values of $\left\{p_{i}\right\}$.

3. Compute matrices $\mathbf{V}_{\mathbf{0}}, \mathbf{U}_{\mathbf{1}}, \ldots, \mathbf{U}_{\mathbf{k}}, \mathbf{A}, \mathbf{B}$ and solve the optimization problem (15) by finding maximal eigenvalue $S_{\max }$ and eigenvector $\Delta \mathbf{p}_{\mathbf{n}}$ of (16)

4. Use the Linesearch strategy to find $\alpha_{n}$ maximizing $S_{\max }\left(\mathbf{p}_{\mathbf{n}}+\alpha_{n} \Delta \mathbf{p}_{\mathbf{n}}\right) \equiv S_{n+1}$

5. Update parameters: $\mathbf{p}_{\mathbf{n}+\mathbf{1}}=\mathbf{p}_{\mathbf{n}}+\alpha_{n} \Delta \mathbf{p}_{\mathbf{n}}$ and a new object location $\mathbf{x}_{\mathbf{n}+\mathbf{1}}=\mathbf{P}\left(\mathbf{x}_{\mathbf{n}}, \mathbf{p}_{\mathbf{n}+\mathbf{1}}\right)$.

6. If $\left|\alpha_{n} \Delta \mathbf{p}_{\mathbf{n}}\right|<\varepsilon_{1}$ and/or $\left|S_{n+1}-S_{n}\right|<\varepsilon_{2}$ then stop; else go to step 1 for a next iteration $n=n+1$.

If the template consists of two regions, $\mathrm{T}_{0}=T_{1} \mathrm{U} T_{2}$, there is an analytic solution of the eigenproblem (16) that does not require iterations. In this case the matrices $\mathbf{A}, \mathbf{B}$ are $\mathbf{A}=\mathbf{V}_{\mathbf{0}}-\mathbf{U}_{\mathbf{0}}, \mathbf{B}=\mathbf{V}_{\mathbf{0}}-\mathbf{U}_{\mathbf{1}}-\mathbf{U}_{\mathbf{2}}$. They are related by $\mathbf{A}-\mathbf{B}=a \mathbf{w} \mathbf{w}^{T}$, derived from the definition of $\mathbf{U}_{\mathbf{i}}(14)$, where $a=n_{1} n_{2} / n_{3}$ and

$$
\mathbf{w}=\frac{1}{n_{1}} \sum_{(x, y) \in T_{1}} \mathbf{f}(x, y)-\frac{1}{n_{2}} \sum_{(x, y) \in T_{2}} \mathbf{f}(x, y)
$$

The vector $\mathbf{w}$ is the linearized contrast between regions $T_{1}$ and $T_{2}$. The solution of (16) is given by the following statement.

Statement: The largest eigenvalue of the eigenproblem (16) and the corresponding eigenvectorare:

$$
\begin{gathered}
\Delta \mathbf{p}_{\max }=\mathbf{B}^{-1} \mathbf{w} \\
S_{\max }=a \mathbf{w}^{T} \Delta \mathbf{p}_{\max }+1
\end{gathered}
$$

Proof: Consider an equivalent eigenvalue problem $(\mathbf{A}-\mathbf{B}) \mathbf{x}=a \mathbf{w} \mathbf{w}^{T} \mathbf{x}=\lambda \mathbf{B x}$, having the same eigenvectors as (16) and eigenvalues transformed as $\quad \lambda=S-1$. Using Cholecky decomposition $\mathbf{B}=\mathbf{L} \mathbf{L}^{T}$, where $\mathbf{L}$ is a bottom-triangular matrix, and introducing a vector transformation $\mathbf{y}=\mathbf{L}^{T} \mathbf{x}$, we obtain another equivalent eigenvalue problem $\mathbf{w}_{\mathbf{1}} \mathbf{w}_{\mathbf{1}}{ }^{T} \mathbf{y}=\lambda \mathbf{y}$, where $\mathbf{w}_{\mathbf{1}}=\mathbf{L}^{-1} \mathbf{w}$. One-rank matrix $\mathbf{w}_{\mathbf{1}} \mathbf{w}_{\mathbf{1}}{ }^{T}$ whose size is $(k+1) \times(k+1)$ has $k$-dimensional eigenspace corresponding to $\lambda_{1}=0$. The vectors $\mathbf{y}_{1}, \ldots, \mathbf{y}_{\mathbf{k}}$ from this eigenspace satisfy $\mathbf{w}_{1}{ }^{T} \mathbf{y}_{\mathbf{i}}=0$. The remaining eigenvector $\mathbf{y}_{\mathbf{k}+\mathbf{1}}$ corresponding to $\lambda_{2} \neq 0$ can be found from the orthogonality condition $\mathbf{y}_{\mathbf{k}+1}{ }^{T} \mathbf{y}_{\mathbf{i}}=0$. Therefore, $\mathbf{y}_{\mathbf{k}+1}=\mathbf{w}_{1}$, from which $\mathbf{x}_{\mathbf{k}+1}=\mathbf{B}^{-1} \mathbf{w}$. Substituting $\mathbf{x}_{\mathbf{k}+1}$ into the eigenvalue equation, we obtain $a \mathbf{w w}^{T} \mathbf{B}^{-1} \mathbf{w}=\lambda \mathbf{B} \mathbf{B}^{-1} \mathbf{w}$, from which $\lambda_{2}=a \mathbf{w}^{T}$ $\mathbf{B}^{-1} \mathbf{W}$. $\mathbf{B}$ is positive-definite; therefore, $\lambda_{2}>0$ and the largest eigenvalue of the problem (16) is $S_{\text {max }}=\lambda_{2}+1=a \mathbf{w}^{T} \Delta \mathbf{p}_{\max }+1$.

The adaptive subpixel STM method (15),(18),(19) has the form similar to geometrically-adaptive subpixel correlation coefficient between images, derived in [14]. Using the 
obtained results, step 3 of the ASSTM algorithm is implemented as follows: 3.1) Compute matrices $\mathbf{V}_{\mathbf{0}}, \mathbf{U}_{\mathbf{1}}, \mathbf{U}_{\mathbf{2}}, \mathbf{B}$ and vector $\mathbf{w} ; 3.2$ ) Solve the system $\mathbf{B} \Delta \mathbf{p}_{\mathbf{n}}=\mathbf{w}$ by Cholecky decomposition; and 3.3) Find the eigenvalue (19).

\section{Experiments}

The presented methods have been applied to a variety of object detection scenarios and tasks, including target detection in remote sensing images, hand, face and facial feature detection, and detection of camera calibration pattern and its structure.
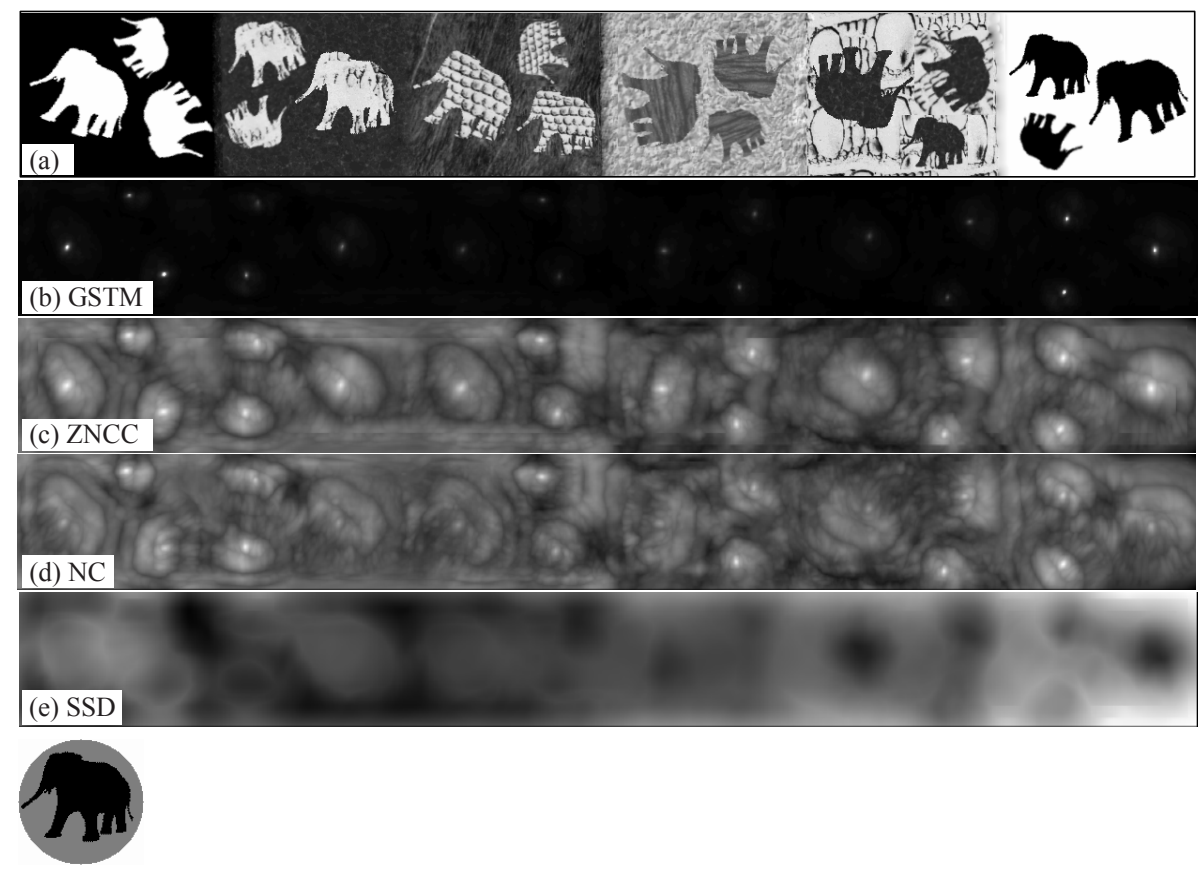

(f)

Fig. 4. Testing the TM methods in the synthetic data. (a) Test image combined from binary images and textured objects on textured backgrounds; (b) GSTM matching score map: large distinctive peaks indicate object locations. (c) Correlation coefficient map: well-recognizable peaks indicate object locations, background matching score values are also large; (d) Normalized contrast map, some object location peaks are less prominent than in ZNCC method; (e) SSD score map; SSD method is not invariant to contrast inversion, so the object locations are defined by the maxima and minima; also, the score map is very smooth (resulting in low accuracy of localization) as there is no local normalization. (f) Two-region template used in all the template matching methods.

For the first experiment, shown in Fig.4, an object was filled by different textures, arbitrarily rotated, and scaled by three factors $1,1.25$ and $1.25^{2}$. The resulting images were placed on textured backgrounds and, together with uniform black-and-white and inverted images, were combined into a single test image (Fig.4a). The two-region template, shown 
in Fig.4f, was used to detect all 18 objects in the test image, using the following methods: 1) GSTM followed by AS STM; 2) TM by zero-mean normalized cross-correlation (ZNCC); 3) TM by normalized contrast (NC), similar to [1]; and 4) TM by the sum of squared differences (SSD). In all these methods, the discrete steps of $10^{\circ}$ for rotation and of 1.25 for scale were used to transform the template and select the largest matching score in each pixel location. The absolute value of the correlation coefficient was used in the ZNCC method to handle inverse contrast. The following matching score was used in the NC method: $\left|m_{1}-m_{2}\right| / \sigma_{0}$. In the ZNCC and SSD methods, the grayscale template was obtained from Fig. 4 b by setting 255 in the gray region and 0 in the black region. Both the GSTM and NC methods were implemented using integral lines, and they performed $~ 50$ times faster than ZNCC or SSD. The following observations can be made comparing the matching score maps (Fig.4b-e) and absolute numerical values of their peaks (Table1): 1) GSTM produced very large and distinctive peaks (this fact becomes more evident after taking ratio of a peak to the background). 2) The peak values can be further improved by AS STM adjusting the object transfor-mation; 3) ZNCC produced similar results, but the peak values are very close to the background matching scores; 4) NC performs worse than both GSTM and ZNCC, as many peaks become undistinguishable from the background; 5) SSD does not solve the problem at all, because: a) it is not invariant to contrast inversion; b) as there is no intensity normalization, the score mapis smooth, resulting in low localization accuracy.

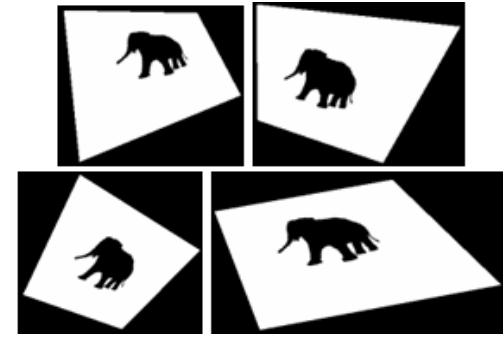

(a)

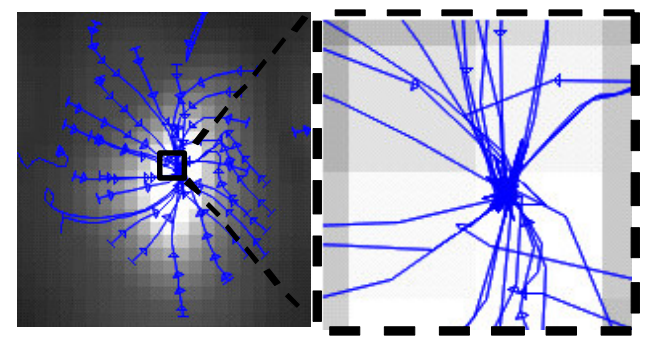

(b)

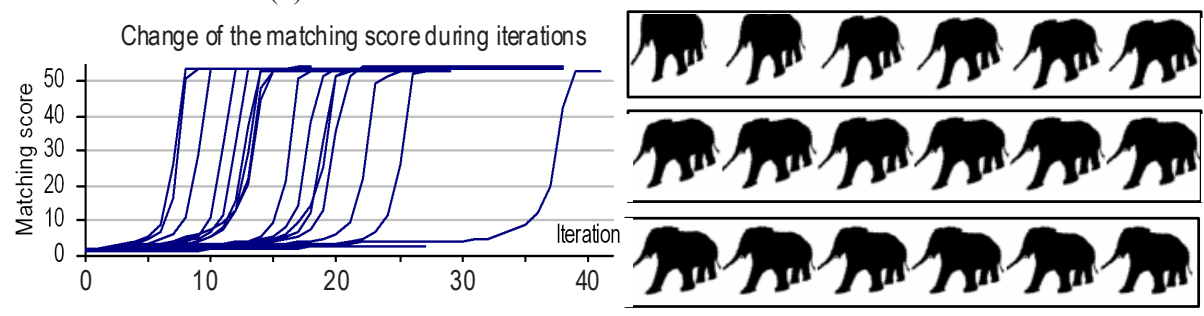

(c)

(d)

Fig. 5. Testing the Adaptive Subpixel STM method in synthetic data. (a) Examples of the perspective transformation of the test image; (b) Trajectories of the image patch center during iterations. Initial positions are selected in the vicinity of matching score peak; almost all trajectories converge to the same subpixel position, which is visible in the zoomed view; (c) Graphs of the matching score along the image patch trajectories. Starting from low values the matching score converges to the true large value, indicating perfect fit of the image data; (d) Example of image patch transformation along one of the trajectories. In this example, the lower-bottom image from figure (a), whose initial orientation estimated by GSTM has been compensated, iteratively transformed so that at the final $18^{\text {th }}$ iteration it coincides with the template (Fig.4f). 
Table 1. Numerical values of the matching score peaks corresponding to 12 objects from Fig. $4 \mathrm{a}$ out of 18. Object counting starts from the leftmost object in Fig.4a. Average matching score, computed from the entire score map, is shown in the last column "Bakground". The last row shows improvement of the matching score after applying ASSTM at the object locations.

\begin{tabular}{|c|c|c|c|c|c|c|c|c|c|c|c|c|c|}
\hline Method \ Object & 1 & 2 & 3 & 4 & 5 & 6 & 7 & 8 & 9 & 10 & 11 & 12 & Backgr. \\
\hline$\overline{Z N C C}$ & 0.98 & 0.95 & 0.97 & 0.91 & 0.91 & 0.93 & 0.81 & 0.83 & 0.80 & 0.87 & 0.88 & 0.87 & 0.34 \\
\hline NC & 19.05 & 28.87 & 23.74 & 27.80 & 22.35 & 18.10 & 15.71 & 25.39 & 19.57 & 17.04 & 26.83 & 21.43 & 9.21 \\
\hline GSTM & 16.44 & 6.02 & 13.41 & 6.09 & 5.40 & 6.29 & 3.07 & 3.75 & 2.90 & 4.70 & 4.39 & 3.92 & 1.21 \\
\hline AS STM & 19.07 & 12.83 & 16.87 & 6.23 & 6.03 & 6.71 & 3.20 & 4.09 & 3.17 & 5.33 & 5.11 & 4.84 & 1.21 \\
\hline
\end{tabular}

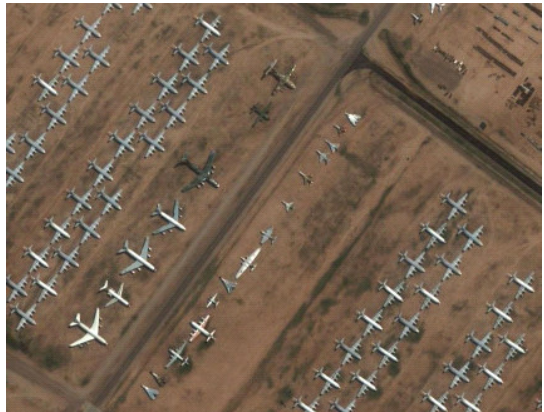

(a)

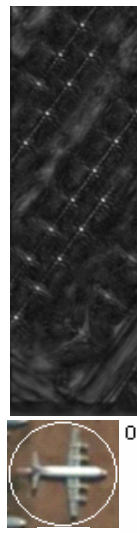

(d)

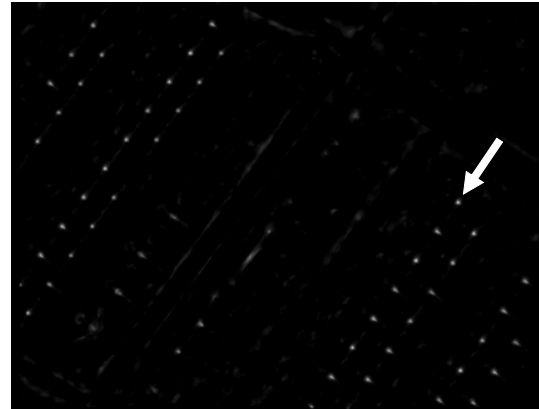

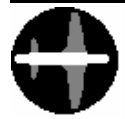

(b)

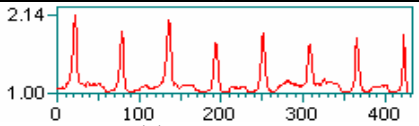

(c)

Fig. 6. Template matching in an aerial image. (a) Test image; (b) Three-region template for GSTM; (c) GSTM matching score map; (d) Template for ZNCC; (e) ZNCC matching score map. The arrows in (c) and (d) show the starting points and directions of the 1D-profiles.

In the second experiment (Fig.5), we generated 100 random perspective transformations of the test image (Fig.5a), and applied the combination GSTM+AS STM with the same template (Fig.4b) to recover the transformation parameters. GSTM was used to roughly estimate object rotation, then AS STM started 30 times from different locations within the vicinity of the matching score peak (Fig.5b). The parameters obtain- ed were compared to ground truth parameters by computing the correlation coefficient 


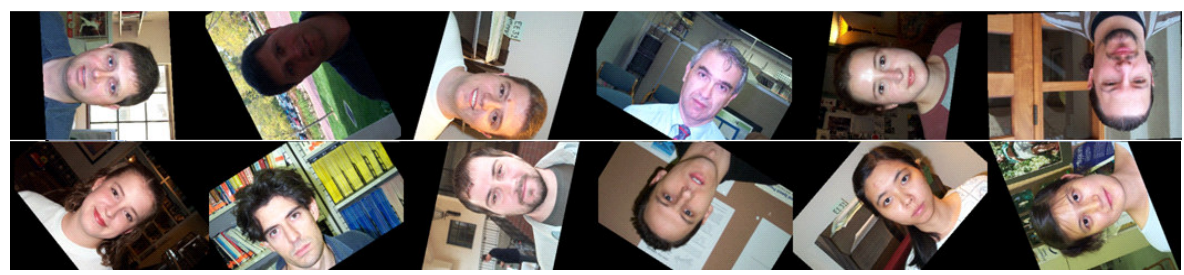

(a)

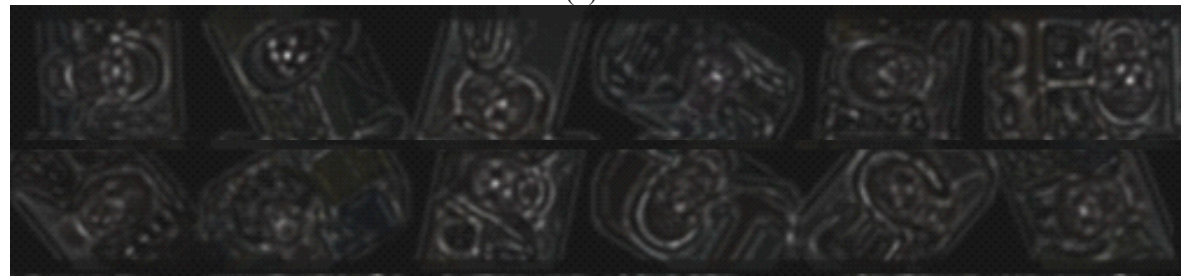

(b)

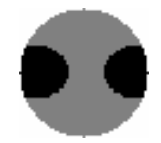

(c)

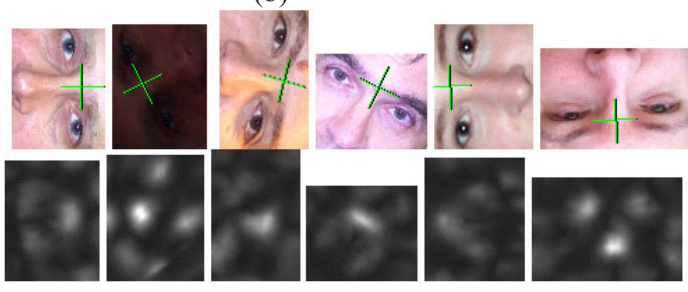

(d)

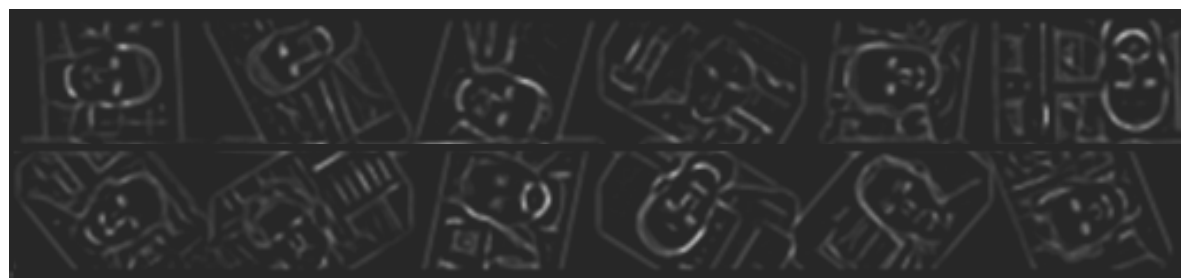

(e)

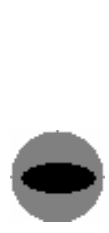

(f)
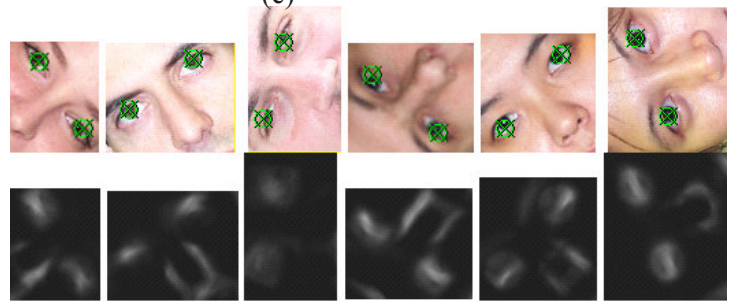

(g)

Fig. 7. STM application to facial feature detection. (a) 12 face images from the Caltech database (http://www.vision.caltech.edu/html-files/archive.html) rotated by random angle; (b) Matching score map resulted from matching with the template in (c); (c) Template for detecting the "between eyes" region; (d) Close-ups of the first 6 images from (a) and corresponding matching score maps from (b). The crosses indicate matching score local maxima; orientation of the crosses corresponds to orientation of the template giving the best matching score; (e) Matching score map resulted from matching with the template in (f); (f) Template for detecting eyes; (g) Close-ups of the images 7-12 from (a) and corresponding matching score maps from (e). The markers indicate matching score local maxima. 
(cosine of the angle between 8-element vectors). In all tests (except for a few cases of distant start), this correlation coefficient was about 1 , indicating high accuracy of estimated parameters. This was also confirmed by high matching score values (Fig.5c). Also, the accuracy of the final location of the image patch was computed. The average distance between the ground truth value and obtained object location and its standard deviation were 0.07 and 0.11 pixels respectively. This experiment confirms the high accuracy of the method and its robustness to initial approximation of object location.

Fig.6 and Fig.7 show two applications where the STM method may be useful. In Fig.6, the method is used to detect airplanes in an aerial image. It was found that the three-region template from Fig.6b gives higher matching scores than a two-region template, in which one region outlines an entire airplane shape. The matching score map clearly shows locations of the object, which can be obtained by searching for local maxima. ZNCC method (Fig.6e) applied using a template taken from the image (Fig.6d), produces similar but more noisy output. Fig.7 demonstrates how GSTM can be used for face and facial feature detection. Two templates were designed to detect eyes and the "between eyes" region (Fig.7c,f), and matching score maps were computed (Fig. $7 \mathrm{~b}, \mathrm{e})$. Taking into account that eye regions are usually darker than surrounding skin, we used filters (4) to remove opposite situation. The detailed results in Fig.7d,g show that the method reliably detected the required facial features.

\section{Conclusions and Future Work}

We have presented novel extensions of the Statistical Template Matching framework that can now be efficiently used to match geometrically transformed objects. Two main contributions of this paper are: 1) integral lines technique for computing the matching score with number of operations linearly dependent on template size; 2) a novel adaptive subpixel refinement method that reduces the problem of transformation parameter estimation to a standard eigenvalue problem.

The proposed methods can also be used to generalize rapid object detection framework [1] to non-Haar-like features, features of complex shape, and arbitrarily oriented features.

Future work will investigate how real image patches can be used to create the simplified multi-region templates. One possible way is to quantize the image into a few levels. We would also like to perform a detailed, quantitative comparison of the STM method with standard template matching methods on real-world data.

\section{References}

1. Viola, P., Jones, M.: Rapid object detection using a boosted cascade of simple features. IEEE CVPR, 511-518 (2001)

2. Sibiryakov, A., Bober, M.: A Method of Statistical Template Matching and Applications to Face and Facial Feature Detection, WSEAS Trans. on Information Science and Applications (September 2005)

3. Jain, A., Zhong, Y., Lakshmanan, S.: Object Matching Using Deformable Templates. IEEE TPAMI 18(3), 267-278 (1996) 
4. Yoshimura, S., Kanade, T.: Fast template matching based on the normalized correlation by using multiresolution eigenimages. In: IEEE/RSJ/GI Int. Conf. on Intelligent Robots and Systems (IROS 1994), vol. 3, pp. 2086-2093 (1994)

5. Jones, M., Viola, P.: Fast Multi-view Face Detection. IEEE CVPR (June 2003)

6. Lienhart, R., Maydt, J.: An extended set of Haar-like features for rapid object detection. In: ICIP 2002, vol. 1, pp. 900-903 (2002)

7. Messom, C.H., Barczak, A.L.: Fast and Efficient Rotated Haar-like Features using Rotated Integral Images. In: Australasian Conf. on Robotics and Automation (2006)

8. Golub, G., Van Loan, C.: Matrix computations. Johns Hopkins University Press, Baltimore (1996), ISBN: 0-8018-5414-8

9. Gould, N., Leyffer, S.: An introduction to algorithms for nonlinear optimization. In: Blowey, J.F., Craig, A.W., Shardlow, T. (eds.) Frontiers in Numerical Analysis, pp. 109197. Springer, Berlin (2003)

10. Lucas, B., Kanade, T.: An iterative image registration technique with an application to stereo vision. In: Proc. of Imaging understanding workshop, pp. 121-130 (1981)

11. Zitova, B., Flusser, J.: Image Registration Methods: a Survey. Image and Vision Computing 24, 977-1000 (2003)

12. Choi, M.-S., Kim, W.-Y.: A novel two stage template matching method for rotation and illumination invariance. Pattern recognition 35(1), 119-129 (2002)

13. Tanaka, K., Sano, M., Ohara, S., Okudaira, M.: A parametric template method and its application to robust matching. IEEE CVPR, 620-627 (2000)

14. Zheltov, S., Sibiryakov, A.: Adaptive Subpixel Cross-Correlation in a Point Correspondence Problem. In: Gruen, A., Kuhbler, O. (eds.) Optical 3D Measurement Techniques, Zurich, September 29 - October 2, pp. 86-95 (1997) 